\title{
STABLE EXTENSIONS OF HOMEOMORPHISMS ON THE PSEUDOARC
}

\author{
JUDY KENNEDY
}

\begin{abstract}
We prove the following:
THEOREM. If $P^{\prime}$ is a proper subcontinuum of the pseudoarc $P, h^{\prime}$ is a homeomorphism from $P^{\prime}$ onto itself, and $\Theta$ is an open set in $P$ that contains $P^{\prime}$, then there is a homeomorphism $h$ from $P$ onto itself such that $h \mid P^{\prime}=h^{\prime}$ and $h(x)=x$ for $x \notin \Theta$.
\end{abstract}

A pseudoarc can be characterized as a chainable, hereditarily indecomposable continuum. Although the main result here extends a result of W. Lewis $[\mathbf{L W}]$ and answers in the affirmative a question he asked in that same paper, Stable homeomorphisms of the pseudoarc, this paper was motivated by a conversation the author had with Professor Marcy Barge, who works in the area of topological dynamics. Professor Barge is interested in knowing what sort of self-homeomorphisms the pseudoarc admits, and, in particular, what sort of homeomorphisms it admits with the property that some of the points of the space have periodic orbits. That pseudoarcs admit many such homeomorphisms will follow from a much earlier result of G. Lehner [LG] and the result here. It will also follow, by combining the theorem proven here, G. Lehner's theorem, and a theorem in $[\mathbf{K J}]$, that any continuum with the property that all its proper nondegenerate subcontinua are pseudoarcs admits many such homeomorphisms. Examples of continua with this property include pseudocircles [B], pseudosolenoids $[\mathbf{F}]$, some examples due to $\mathrm{T}$. Ingram $[\mathbf{I}]$ and $\mathrm{H}$. Cook $[\mathbf{C}]$, as well as the pseudoarc itself.

We will prove the following theorem:

THEOREM. If $P^{\prime}$ is a proper, nondegenerate subcontinuum of the pseudoarc $P$, $P^{\prime} \subseteq o$, an open set in $P$, and $h$ is a homeomorphism from $P^{\prime}$ onto itself, then $h$ can be extended to a homeomorphism $\hat{h}$ of $P$ onto itself such that $\hat{h}(x)=x$ for $x \notin o$.

A continuum is indecomposable if no proper subcontinuum of it has interior, and it is hereditarily indecomposable if each subcontinuum is indecomposable. A chain is a collection of sets $C=\left\{C_{0}, C_{1}, \ldots, C_{n}\right\}$ such that $C_{i} \cap C_{j} \neq \varnothing$ if and only if $|i-j| \leq 1$. A continuum is chainable if it has open chain covers of arbitrarily small mesh. As mentioned previously, the pseudoarc $P$ is a hereditarily indecomposable, chainable continuum [KB]. This continuum also has the fixed point property $[\mathbf{H}]$.

Received by the editors June 9,1987 . This research was presented at the Annual Spring Topology Conference, March 1, 1987, University of Alabama at Birmingham. (This conference was supported by the NSF.).

1980 Mathematics Subject Classification (1985 Revision). Primary 54F20, 54F50.

Key words and phrases. Continuum, pseudoarc, chainable, pseudocircle, hereditarily indecomposable, stable homeomorphism, extensions of homeomorphisms. 
The elements of a chain are called links. A chain $C=\left\{C_{0}, \ldots, C_{n}\right\}$ is taut whenever $C_{i} \cap C_{j} \neq \varnothing$ if and only if $\bar{C}_{i} \cap \bar{C}_{j} \neq \varnothing$. A chain covers a set $A$ essentially if there is a continuum $Q$ contained in $A$ such that each link contains a point of $Q$ not in the closure of any other link. An open set $o$ in a space $X$ is regular if Int $\bar{o}=o$. In the discussion that follows we will assume that our open chain covers are regular, taut, and essential.

If $B$ is a collection of sets, then $B^{*}$ denotes the union of the sets in $B$. If $C$ is a collection of sets, then $C$ is an amalgamation of $B$ if $B^{*}=C^{*}$ and each set in $C$ is the union of some sets in $B$. If the closure of each set in $B$ is a subset of a set in $C$, then $B$ is said to closure refine $C$.

If $X$ is a topological space, $H(X)$ will denote the collection of homeomorphisms from $X$ onto $X$.

Suppose that $d$ is a metric on the pseudoarc $P$ that is compatible with its topology. Throughout the paper we will take distances with respect to this metric $d$, and for $x \in P, \varepsilon>0, D_{\varepsilon}(x)=\{y \in P \mid d(x, y)<\varepsilon\}$. Also, if $H$ and $K$ are closed subsets of the pseudoarc, $d(H, K)=\min \{d(x, y) \mid x \in H, y \in K\}$. If $H=\{x\}$, we will write $d(x, K)$ for $d(\{x\}, K)$.

If $C=\left\{C_{0}, \ldots, C_{n}\right\}$ is an open chain in a space $X$ (which does not necessarily cover $X)$, then for $c \in C$,

$$
i(c, C)=\left\{y \in c \mid y \notin \bar{c}^{\prime} \text { for } c^{\prime} \in C-\{c\}\right\},
$$

and

$$
j(c, C)=\left\{y \in \bar{c} \mid y \notin \bar{c}^{\prime} \text { for } c^{\prime} \in C-\{c\}\right\} .
$$

We will say that $C$ has the end link exit property if $\partial C^{*} \subseteq j(c, C)$ where $c$ is one of the end links of $C$. If $\partial C^{*} \subseteq j(c, C) \cup j\left(c^{\prime}, C\right)$ where $c$ and $c^{\prime}$ are the end links of $C$, then $C$ has the weak end link exit property. If $C$ is an open chain with the end link exit property, $C=\left\{c_{0}, \ldots, c_{m}\right\}$ and $\partial C^{*} \subseteq j\left(c_{0}, C\right)$, then $\mathbf{C}(C)=\left\{c_{0} \cup\left(\bar{c}_{0} \cap\right.\right.$ $\left.\left.\partial C^{*}\right), c_{1}, \ldots, c_{m}\right\}$. If $C=\left\{c_{0}, \ldots, c_{m}\right\}$ is an open chain cover of $\bar{o}$, considered as space, where $o$ is an open set in $X$, and $\partial_{o} \subseteq j\left(c_{0}, C\right), \mathbf{I}(C)=\left\{\operatorname{Int}\left(c_{0}\right), c_{1}, \ldots, c_{m}\right\}$. Also, $C[k, l]=\left\{c_{k}, c_{k+1}, \ldots, c_{l}\right\}$.

We will denote the nonnegative integers by $\mathbf{N}$. If $m, n \in \mathbf{N}, m<n$, let $[m, n]=$ $\{m, m+1, \ldots, n\}$. A function $f:\left[m^{\prime}, n^{\prime}\right] \rightarrow[m, n]$ is called a (light) pattern provided $|f(i+1)-f(i)| \leq 1\left(|f(i+1)-f(i)|=1\right.$, respectively) for $i=m^{\prime}, \ldots, n^{\prime}-1$. (The symbol $\rightarrow$ indicates that $f$ is an onto function.) If $V=\left\{V_{m^{\prime}}, V_{m^{\prime}+1}, \ldots, V_{n^{\prime}}\right\}$ and $U=\left\{U_{m}, U_{m+1}, \ldots, U_{n}\right\}$ are chain covers of the compactum $X$, and $f:\left[m^{\prime}, n^{\prime}\right] \rightarrow$ $[m, n]$ is a pattern, we will say that $V$ follows pattern $f$ in $U$ provided $\bar{V}_{i} \subseteq V_{f(i)}$ for each $i \in\left[m^{\prime}, n^{\prime}\right]$. We call $f$ a pattern on $U$. If $A \subseteq U$, we will say that $f$ prefers $A$ if whenever $V_{i} \in V$ and $\bar{V}_{i} \subseteq U_{j} \in A$, for some $j$, then $U_{f(i)} \in A$.

The chain $C$ properly covers the chain $D$ if $D$ closure refines $C$ and for every $c \in C$ there is some $d$ in $D$ such that $\bar{d} \subseteq c$. Suppose that $C=\{c(0), \ldots, c(m)\}, D=$ $\{d(0), \ldots, d(m)\}$ and $E=\{e(0), \ldots, e(n)\}$ are open chains covering the pseudoarc, $X$ is a collection of links of $C$ and $f:[0, n] \rightarrow[0, m]$ is a pattern that $E$ follows in $C$. Then $(E, f)$ is compatible with $D$ relative to $(C, X)$ provided that:

(1) each of $C$ and $D$ is an amalgamation of $E$;

(2) $C(f(j)) \in X$ if $\overline{E(j)} \subseteq X^{*}$;

(3) $C(i)=D(i)$ for every $C(i) \in X$; 
(4) for each subchain $\tilde{E}$ of $E$ with $\{C(f(k)) \mid E(k) \in \tilde{E}\} \cap X \neq \varnothing$, $\tilde{E}$ is properly covered by $\{D(f(k)) \mid E(k) \in \tilde{E}\}$.

We will make the following notational conventions: chains will be denoted with upper-case letters, and possibly additional symbols and links of chains with the associated lower-case letters, associated symbols, and link numbers. So, for example,

$$
\begin{aligned}
C_{1} & \equiv\{c(1,0), \ldots, c(1, m)\} \equiv C_{1}[0, m] \\
\tilde{D}_{2} & \equiv\{\tilde{d}(2, k), \ldots, \tilde{d}(2, l)\} \equiv \tilde{D}_{2}[k, l] \\
F & \equiv\{f(1), \ldots, f(n)\} \equiv\left\{f_{1}, \ldots, f_{n}\right\} \equiv F[1, n] .
\end{aligned}
$$

The preceding is largely the terminology and notation of $[\mathbf{O T}]$ and $[\mathbf{L W}]$. We will also make use of the following result from [LW].

THEOREM LW (THEOREM 6 OF [LW]). Let $C_{0}, D_{0}$, and $C_{1}$ be chains covering the pseudoarc, with $X$ a nonempty collection of links of $C_{0}$ and $f_{1}$ a pattern such that $\left(C_{1}, f_{1}\right)$ is compatible with $D_{0}$ relative to $\left(C_{0}, X\right)$. Then for each $\varepsilon>0$ there exists a chain $E$ of mesh less than $\varepsilon$, a pattern $f_{2}$, and a chain $D_{1}$ covering $P$ such that $D_{1}$ follows the pattern $f_{1}$ in $D_{0}$, and $\left(E, f_{2}\right)$ is compatible with $C_{1}$ relative to $\left(D_{1},\left\{d(1, j) \mid c\left(0, f_{1}(j)\right) \in X\right\}\right)$.

We will also need the following theorems due to G. R. Lehner [LG] and J. Krasinkiewicz and P. Minc [OT], respectively.

THEOREM LG. Suppose $H_{1,1}, H_{1,2}, \ldots, H_{1, n}$ are disjoint subcontinua of the pseudoarc $P$ and that $P$ is irreducible between each pair of them. Suppose $T$ is a homeomorphism of $\bigcup_{i=1}^{n} H_{1, i}$ onto $\bigcup_{i=1}^{n} H_{2, i}$ where $H_{2,1}, H_{2,2}, \ldots, H_{2, n}$ are subcontinua of $P$ such that $P$ is irreducible between each pair of them. Then $T$ can be extended to a homeomorphism of $P$ onto $P$.

THEOREM KM. Let $X$ be a compactum. Then the following are equivalent:

(I) $X$ is hereditarily indecomposable;

(II) for every pair of disjoint closed subsets $A$ and $B$ of $X$ and for every pair of neighborhoods $U$ of $A$ and $V$ of $B$, there exist closed subsets $X_{0}, X_{1}$ and $X_{2}$ of $X$ such that

(1) $X=X_{0} \cup X_{1} \cup X_{2}$,

(2) $A \subseteq X_{0}$ and $B \subseteq X_{2}$,

(3) $X_{0} \cap X_{2}=\varnothing$,

(4) $X_{0} \cap X_{1} \subseteq V$ and $X_{1} \cap X_{2} \subseteq U$.

A sequence $V=V_{1}, V_{2}, \ldots$ of open chain covers of $P$ is said to be a defining sequence for $P$ if $\lim _{i}$ mesh $V_{i}=0$ and, for each $i, V_{i}$ is an amalgamation of $V_{i+1}$. (Please recall that "open chain cover" really means "open taut essential regular chain cover.") We will say that a set is $V_{j}$-open if it is a finite union of links of $V_{j}$ for some $j$.

LEMMA 2. Suppose that $P^{\prime}$ is a proper, nondegenerate subcontinuum of $P$, and $C=\left\{c_{0}, \ldots, c_{m}\right\}$ is an open chain cover of $P^{\prime}$, considered as space, of mesh less than $\varepsilon$. There is an open (in $P$ ) chain $D=\left\{d_{0}, \ldots, d_{m}\right\}$ that covers $P^{\prime}$, has mesh less than $\varepsilon$, has the end link exit property with $\partial D^{*} \supseteq j\left(d_{0}, D\right)$, and has the property that $d_{i} \cap P^{\prime}=c_{i}$ for each $i \in[0, m]$. 
ProOF. Suppose $P^{\prime}$ is irreducible between $p \in i\left(c_{0}, C\right)$ and $g \in i\left(c_{m}, C\right)$. Suppose that $\varepsilon^{\prime}=\operatorname{mesh} C$ and $V_{1}, V_{2}, \ldots$ is a defining sequence for $P$ such that if

$$
V_{i}^{\prime}=\left\{v \cap P^{\prime} \mid \bar{v} \cap P^{\prime} \neq \varnothing, v \in V_{i}\right\}
$$

then $V_{1}^{\prime}, V_{2}^{\prime}, \ldots$ is a defining sequence for $P^{\prime}$. Also, we may assume that $g \in$ $i\left(v\left(i, n_{i}\right), V_{i}\right)$, where $v\left(i, n_{i}\right)$ is the last link of $V_{i}$. (It can easily be checked that such a sequence exists.) Choose $\sigma>0$ so that $\sigma<\min \left\{d\left(\bar{c}_{i}, \bar{c}_{j}\right)|| i-j \mid>1, i, j \in[0, m]\right\}$. There is an integer $p(1)$ so that $V_{p(1)}^{\prime}$ closure refines $C$, and if $p \geq p(1), V_{p}$ has mesh less than $\left(\varepsilon-\varepsilon^{\prime}\right) / 4$ and $\sigma / 4$. Choose $\delta<\varepsilon-\varepsilon^{\prime}$. We want for $p$ to be in the first link of $V_{p(1)}$. Otherwise apply Theorem KM as follows: Suppose $v\left(p(1), a_{p(1)}\right)$ is the first link of $V_{p(1)}$ that intersects $P^{\prime}$. Let $B=\partial V_{p(1)}\left[a_{p(1)}, n_{p(1)}\right]^{*}$. Suppose $o$ is an open set such that

$$
\begin{aligned}
B \subseteq & o \subseteq \bar{o} \subseteq v\left(p(1), a_{p(1)}\right) \cup v\left(p(1), a_{p(1)}-1\right) \\
& -\left(\left\{\overline{v \in V_{p(1)} \mid v \neq v\left(p(1), a_{p(1)}\right) \text { and } v \neq v\left(p(1), a_{p(1)}-1\right)}\right\}^{*} \cup P^{\prime}\right) .
\end{aligned}
$$

Without loss of generality, assume $p \in i\left(v(p(1), b), V_{p(1)}\right)$ and that $u$ is open such that $p \in u \subseteq \bar{u} \subseteq i\left(v(p(1), b), V_{p(1)}\right)$. There are closed sets $X_{0}, X_{1}$, and $X_{2}$ such that

(1) $X_{0} \cup X_{1} \cup X_{2}=V_{p(1)}\left[a_{p(1)}, n_{p(1)}\right]^{*} \cup \bar{o}$;

(2) $B \subseteq X_{0}$ and $p \in X_{2}$; and

(3) $X_{0} \cup X_{1} \subseteq u$ and $X_{1} \cap X_{2} \subseteq o$.

Now form $\tilde{V}_{p(1)}$ as follows: Let $X=X_{1} \cup X_{2}$. There is a chain $T_{0}$ such that $\left\{v \cap \operatorname{Int}\left(X_{0}\right) \mid v \in V_{p(1)}\left[a_{p(1)}, n_{p(1)}\right], v \cap X_{0} \neq \varnothing\right\}$ is an amalgamation of $T_{0}$, and a chain $T_{1}$ such that $\left\{v \cap \operatorname{Int}(X) \mid v \in V_{p(1)}\left[a_{p(1)}, n_{p(1)}\right], v \cap X \neq \varnothing\right\}$ is an amalgamation of $T_{1}$, and $T_{0}$ and $T_{1}$ are open chains such that

(4) $T_{0}^{*} \cup T_{1}^{*}=V_{p(1)}\left[a_{p(1)}, n_{p(1)}\right]^{*}$;

(5) $\tilde{V}_{p(1)}=V_{p(1)}\left[0, a_{p(1)}-1\right] \cup T_{0} \cup T_{1}$ is an open chain cover of $P$ that refines $V_{p(1)}$

(6) $p$ is in the first link of $\tilde{V}_{p(1)}$ whose closure intersects $P^{\prime}$ and is not in the closure of any other link of $\tilde{V}_{p(1)}$; and

(7) $q$ is in the last link of $\tilde{V}_{p(1)}$ and not in the closure of any other link of $\tilde{V}_{p(1)}$;

(8) if $\tilde{V}_{p(1)}^{\#}=\left\{v \in \tilde{V}_{p(1)} \mid \bar{v} \cap P^{\prime} \neq \varnothing\right\}$, then $\partial \tilde{V}_{p(1)}^{\#} \subseteq j\left(\hat{v}, \tilde{V}_{p(1)}^{\#}\right)$, where $\hat{v}$ is the first link in $\tilde{V}_{p(1)}^{\#}$, and $p \in i\left(\hat{v}, \tilde{V}_{p(1)}\right)$. Thus, we can always modify $V_{p(1)}$ so that $p$ is in the first link of $V_{p(1)}^{\prime}$ and $q$ is in the last link of $V_{p(1)}^{\prime}$, etc. In fact, we have actually shown that we could have chosen our sequence $V_{1}, V_{2}, \ldots$ so that $p$ is in the first link of $V_{i}^{\prime}$ and $q$ is in the last link of $V_{i}$. Let us assume that our sequence has this property then, so that we will not have to worry about this again.

For $j \in[0, m]$, let $d_{j}(1)=\left\{v \in V_{p(1)} \mid \varnothing \neq \bar{v} \cap P^{\prime} \subseteq c_{j}\right\}^{*}$. Then $D(1)=$ $\left\{d_{0}(1), \ldots, d_{m}(1)\right\}$ is an open (in $P$ ) chain cover of $P^{\prime}$ of mesh less than $\varepsilon^{\prime}+\delta / 2$. Also, since $D(1)$ is an amalgamation of a subchain of $V_{p(1)}, D(1)$ has the end link exit property with $\partial D(1)^{*} \subseteq j\left(d_{0}(1), D(1)\right)$, and $D^{\prime}(1)=\left\{d \cap P^{\prime} \mid d \in D(1)\right\}$ closure refines $C$.

Let $E=\left\{d_{j}(1) \mid j \in[1, m]\right\}^{*}$ and $\sigma^{\prime}>0$ such that

$$
d\left(P^{\prime}, \partial D(1)^{*}\right)>\sigma^{\prime}, \quad d\left(E, \partial D(1)^{*}\right)>\sigma^{\prime} .
$$


Choose a sequence $p(2), p(3), \ldots$ of increasing integers such that

(1) $p(2)>p(1)$

(2) $V_{p(i)}$ has mesh less than $\sigma^{\prime} / 4$ for $i \geq 2$, and

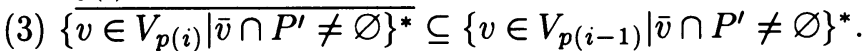

For $j \in[0, m], i \geq 2$, let $d_{j}(i)=\left\{v \in V_{p(i)} \mid \varnothing \neq \bar{v} \cap P^{\prime} \subseteq c_{j}\right\}^{*}$. Then $D(i)=$ $\left\{d_{j}(i) \mid 0 \leq j \leq m\right\}$ is an open (in $P$ ) chain cover of $P^{\prime}$ of mesh less than $\varepsilon^{\prime}+\delta / 2$, and $D(i)$ has the end link exit property. Further, $D^{\prime}(i)=\left\{d \cap P^{\prime} \mid d \in D(i)\right\}$ closure refines $C$. For $j \in[0, m]$ let $d_{j}=\bigcup_{i=1}^{\infty} d_{j}(i)$, and let $D=\left\{d_{0}, \ldots, d_{m}\right\}$. It is clear that $D$ is an open (in $P$ ) cover of $P^{\prime}$ and that $d_{j} \cap P^{\prime}=c_{j}$. Suppose $x$ and $y$ are in $d_{j} \in D$. There are $i$ and $i^{\prime}$ such that $x \in d_{j}(i)$ and $y \in d_{j}\left(i^{\prime}\right)$, and there are $z$ and $z^{\prime}$ in $c_{j}$ such that $d(z, x)<\delta / 4$ and $d\left(z^{\prime}, y\right)<\delta / 4$. Then

$$
d(x, y) \leq d(x, z)+d\left(z, z^{\prime}\right)+d\left(z^{\prime}, y\right)<\delta / 4+\varepsilon^{\prime}+\delta / 4=\varepsilon^{\prime}+\delta / 2<\varepsilon^{\prime}+\delta .
$$

Thus, the mesh of $D$ is less than $\varepsilon^{\prime}+\delta=\varepsilon$.

Suppose that $\bar{d}_{i} \cap \bar{d}_{j} \neq \varnothing$. There is $w \in \bar{d}_{i} \cap \bar{d}_{j}$ and we can find $w^{\prime} \in d_{i}(l)$ for some $l$, and $\hat{w} \in d_{j}\left(l^{\prime}\right)$ for some $l^{\prime}$, such that $d\left(w^{\prime}, w\right)<\sigma / 4$ and $d(w, \hat{w})<\sigma / 4$. There are $p^{\prime}$ and $\hat{p}$ in $c_{i}$ and $c_{j}$, respectively, such that $d\left(w^{\prime}, p^{\prime}\right)<\sigma / 4$ and $d(\hat{w}, \hat{p})<\sigma / 4$. Then $d\left(p^{\prime}, \hat{p}\right) \leq d\left(p^{\prime}, w^{\prime}\right)+d\left(w^{\prime}, \hat{w}\right)+d(\hat{w}, \hat{p})<\sigma$. By the choice of $\sigma$, this means that $|i-j| \leq 1$ and that $D$ is a (taut) chain.

Consider finally $\partial D^{*} . D^{*}=D(1)^{*}$, since

$$
D(1)^{*}=\left\{v \in V_{p(1)} \mid \bar{v} \cap P^{\prime} \neq \varnothing\right\}^{*} \supseteq D(i)^{*} \text { for } i>1
$$

(by the choice of $\sigma^{\prime}$ ). If $x \in \partial D^{*}, x \in \partial D(1)^{*}$. Suppose $x \in \bar{d}_{j}$ for some $j$. There is $x^{\prime} \in D(1)^{*}$ such that $d\left(x^{\prime}, x\right)<\sigma^{\prime} / 4, x^{\prime} \in d_{j}(l)$ for some $l$. If $l>1$, there is $\hat{x} \in c_{j}$ so that $d\left(x^{\prime}, \hat{x}\right)<\sigma^{\prime} / 4$. Then $d(x, \hat{x}) \geq d\left(x, x^{\prime}\right)+d\left(x^{\prime}, \hat{x}\right)<\sigma^{\prime} / 2$, which cannot be because of the choice of $\sigma^{\prime}$. Then $l=1$ and $x^{\prime} \in d_{j}(1)$. But $x^{\prime} \in d_{j}(1)$ only if $j=0$, so we are done.

We will refer to the process of applying Lemma 2 as fattening an open chain in $P^{\prime}(C)$ to obtain an open chain in $P(D)$.

LEMMA 3. Suppose that $P^{\prime}$ is a nondegenerate, proper subcontinuum of $P$ and $C=\left\{c_{0}, \ldots, c_{m}\right\}$ is an open (in $P$ ) chain cover of $P^{\prime}$, with $\partial C^{*} \subseteq j\left(c_{m}, C\right)$, and $P^{\prime}$ is covered essentially by $C$. There is an open chain $D=\left\{d_{0}, \ldots, d_{2 m}\right\}$ such that

(1) $D$ refines $C$,

(2) $\partial D^{*} \subseteq j\left(d_{0}, D\right) \subseteq i\left(c_{m}, C\right)$,

(3) $P^{\prime} \cap d_{m+i}=P^{\prime} \cap c_{i}$ for $i \in[0, m]$, and

(4) $P^{\prime} \cap \bar{d}_{i}=\varnothing$ for $i \in[0, m-1]$.

PrOOF. There is a point $p \in i\left(c_{0}, C\right) \cap P^{\prime}$. Find disjoint open sets $u$ and $v$ such that $p \in u \subseteq \bar{u} \subseteq i\left(c_{0}, C\right)$ and $\partial C^{*} \subseteq v, \bar{v} \cap P^{\prime}=\varnothing, \bar{v} \cap \bar{c}_{i}=\varnothing$ for $i \in[0, m-1]$. If $X=\bar{C}^{*}$, we can apply Theorem 2 of [OT] (which is actually a theorem of Krasinkiewicz and Minc, but is stated in the form we need in [OT]) to find closed sets $X_{0}, X_{1}$, and $X_{2}$ of $X$ such that

(1) $p \in X_{2}$ and $\partial C^{*} \subseteq X_{0}$,

(2) $X=X_{0} \cup X_{1} \cup X_{2}$,

(3) $X_{0} \cap X_{2}=\varnothing$

(4) $X_{0} \cap X_{1} \subseteq u$ and $X_{1} \cap X_{2} \subseteq v$. 
Then $P^{\prime} \subseteq$ Int $X_{2}$. Define $d_{0}=\left(c_{m} \cap X_{0}\right)-\bar{v}$. For $i \in[1, m-1]$, define $d_{i}=$ $c_{m-i} \cap X_{0}$. Define $d_{m}=c_{0}$, and for $i \in[m+1,2 m], d_{i}=c_{i-m} \cap\left(X_{1} \cup X_{2}\right)$. Finally, $D=\left\{d_{0}, \ldots, d_{2 m}\right\}$. We have the chain we wanted.

Given an open, in $P^{\prime}$, chain cover $C=\left\{c_{0}, \ldots, c_{m}\right\}$, we will refer to the process of obtaining the chains (open in $P$ ) that Lemmas 1-3 guarantee us as fattening and doubling $C$.

LEMMA 4. Suppose that $C_{0}=C_{0}\left[0, m_{0}\right], D_{0}=D_{0}\left[0, m_{0}\right]$, and $\hat{C}_{1}=\hat{C}_{1}\left[0, \hat{m}_{1}\right]$ are chains covering the pseudoarc $P$, such that $\hat{C}_{1}$ closure refines $C_{0}$ and $D_{0}, X \subseteq$ $C_{0}, \hat{f}_{1}$ is a pattern, and $\left(\hat{C}_{1}, \hat{f}_{1}\right)$ is compatible with $D_{0}$ relative to $\left(C_{0}, X\right)$. Further, suppose $C_{1}^{\prime}$ is an open chain cover of $P$ so that $C_{1}^{\prime}$ closure refines $\hat{C}_{1}, \hat{C}_{1}$ is an amalgamation of $C_{1}^{\prime}$, and $X^{\prime}=\left\{c \in X \mid c \cap\left(C_{0}-X\right)^{*}=\varnothing\right\}$. If $C_{1}=\left\{\tilde{C}^{*} \mid \tilde{C}\right.$ is a maximal subchain of $C_{1}^{\prime}$ such that for some $\hat{c} \in \hat{C}_{1}, \tilde{C}^{*} \subseteq \hat{c}$ and $\left.i\left(\hat{c}, \hat{C}_{1}\right) \cap \tilde{C}^{*} \neq \varnothing\right\}$, then $C_{1}$ is an open chain cover of $P, C_{1}=C_{1}\left[0, m_{1}\right]$, and $f_{1}:\left[0, m_{1}\right] \rightarrow\left[0, m_{0}\right]$ is a pattern defined by $f_{1}(i)=\hat{f}_{1}(j)$, where $c(1, i) \subseteq \hat{c}(1, j)$ and $c(1, i) \cap i\left(\hat{c}(1, j), \hat{C}_{1}\right) \neq$ $\varnothing$, unless $c\left(0, \hat{f}_{1}(j)\right) \notin X^{\prime}, \overline{c(1, i)} \subset X^{\prime *}$, in which case $f_{1}(i)$ is defined so that $f_{1}$ will prefer $X^{\prime}$, then $\left(C_{1}, f_{1}\right)$ is compatible with $D_{0}$ relative to $\left(C_{0}, X^{\prime}\right)$.

PROOF. This proof is straightforward and will be omitted.

LEMMA 5. Suppose that $P^{\prime}$ is a proper nondegenerate subcontinuum of $P$, and that $p^{\prime} \in P^{\prime}$. Suppose further that (1) $C_{0}=C_{0}\left[0, m_{0}\right], D_{0}=D_{0}\left[0, m_{0}\right]$, and $C_{1}=C_{1}\left[0, m_{1}\right]$ are chains covering $P$ such that

(1) $C_{1}$ closure refines $C_{0}$ and $D_{0}$;

(2) $p^{\prime} \in i\left(c\left(0, m_{0}\right), C_{0}\right) \cap i\left(d\left(0, m_{0}\right), D_{0}\right) \cap i\left(c\left(1, m_{1}\right), C_{1}\right)$;

(3) $\operatorname{mesh} C_{1}<\frac{1}{6} \delta$ where $\delta=\min \left\{d\left(p^{\prime}, e\right) \mid p^{\prime} \notin e, e \in C_{0} \cup D_{0}\right\}$;

(4) $X \subseteq C_{0}$ such that $P^{\prime} \cap \bar{X}^{*}=\varnothing$; and

(5) $f_{1}$ is a pattern with $\left(C_{1}, f_{1}\right)$ compatible with $D_{0}$ relative to $\left(C_{0}, X\right)$.

Then for each $\varepsilon>0$ there exists a chain $D_{2}$ of mesh less than $\varepsilon$, a pattern $f_{2}$, and a chain $D_{1}$ covering $P$ so that $D_{1}$ follows the pattern $f_{1}$ in $D_{0}, p^{\prime} \in i\left(d\left(1, m_{1}\right), D_{1}\right) \cap$ $i\left(d\left(2, m_{2}\right), D_{2}\right)$ and $\left(D_{2}, f_{2}\right)$ is compatible with $C_{1}$ relative to $\left(D_{1}, X_{1}^{\prime}\right)$ where $X_{1}^{\prime}=$ $\left\{c \in X_{1} \mid c \cap\left(C_{1}-X_{1}\right)^{*}=\varnothing\right\}$ and $X_{1}=\left\{c(1, j) \mid c\left(0, f_{1}(j)\right) \in X\right\}$.

ProOF. Suppose $\varepsilon>0$. There are open sets $u, v, w$ such that (1) $\{u, v, w\}$ is an open chain with $c\left(1, m_{1}\right)=u \cup v \cup w$; (2) $p^{\prime} \in w-\overline{u \cup v}$; (3) $\partial\{u, v, w\}^{*}=$ $\partial\left(c\left(1, m_{1}\right)\right) \subseteq \partial u$; and $(4)(\bar{w} \cup \bar{v}) \cap \overline{c\left(1, m_{1}-1\right)}=\varnothing$. Modify $C_{0}$ and $D_{0}$ next: $\hat{C}_{0}=\hat{C}_{0}\left[0, m_{0}+1\right]$ is defined by

$$
\hat{c}(0, i)= \begin{cases}c(0, i) & \text { for } i<m_{0}, \\ \left(c\left(0, m_{0}\right)-w\right) \cup v & \text { for } i=m_{0}, \\ u \cup v \cup w & \text { for } i=m_{0}+1,\end{cases}
$$

and $\hat{D}_{0}=\hat{D}_{0}\left[0, m_{0}+1\right]$ is defined by

$$
\hat{d}(0, i)= \begin{cases}d(0, i) & \text { for } i<m_{0}, \\ \left(d\left(0, m_{0}\right)-w\right) \cup v & \text { for } i=m_{0}, \\ u \cup v \cup w & \text { for } i=m_{0}+1 .\end{cases}
$$


Further, define $\hat{C}_{1}=\hat{C}_{1}\left[0, m_{1}+2\right]$ by

$$
\hat{c}(1, i)= \begin{cases}c(1, i) & \text { for } i<m_{1}, \\ u & \text { for } i=m_{1}, \\ v & \text { for } i=m_{1}+1 \\ w & \text { for } i=m_{1}+2\end{cases}
$$

Then $\hat{C}_{1}$ follows $\hat{f}_{1}$ in $\hat{C}_{0}$ where $\hat{f}_{1}(i)=f_{1}(i)$ for $i<m_{1}, \hat{f}_{1}\left(m_{1}\right)=m_{0}, \hat{f}_{1}\left(m_{1}+1\right)=$ $\hat{f}_{1}\left(m_{1}+2\right)=m_{0}+1$, and $\left(\hat{C}_{1}, \hat{f}_{1}\right)$ is compatible with $\hat{D}_{0}$ relative to $\left(\hat{C}_{0}, X\right)$. Theorem LW allows us to find a chain $\hat{D}_{2}=\hat{D}_{2}\left[0, \hat{m}_{2}\right]$ of mesh less than $\varepsilon$, a pattern $\hat{f}_{2}$, and a chain $\hat{D}_{1}=\hat{D}_{1}\left[0, m_{1}+2\right]$ covering $P$ so that $\hat{D}_{1}$ follows $\hat{f}_{1}$ in $\hat{D}_{0}$ and $\left(\hat{D}_{2}, \hat{f}_{2}\right)$ is compatible with $\hat{C}_{1}$ relative to $\left(\hat{D}_{1},\left\{\hat{d}(1, j) \mid \hat{c}\left(0, \hat{f}_{1}(j)\right) \in X\right\}\right)$. Note that $p^{\prime}$ must be in either $\hat{d}\left(1, m_{1}+1\right)$ or $\hat{d}\left(1, m_{1}+2\right)$, for $\hat{f}_{1}\left(m_{1}+1\right)=$ $\hat{f}_{1}\left(1, m_{1}+2\right)=m_{0}+1, \hat{f}_{1}(i)<m_{0}+1$ for $i<m_{1}+1$, and $p^{\prime} \in i\left(\hat{d}\left(0, m_{0}+1\right), \hat{D}_{0}\right)$. Let $D_{1}=D_{1}\left[0, m_{1}\right]$, where

$$
d(1, i)= \begin{cases}\hat{d}(1, i) & \text { for } i<m_{1}, \\ \bigcup_{j=0}^{2} \hat{d}\left(1, j+m_{1}\right) & \text { for } i=m_{1} .\end{cases}
$$

Then $D_{1}$ follows $f_{1}$ in $D_{0}$. Define $f_{2}^{\prime}:\left[0, \hat{m}_{2}\right] \rightarrow\left[0, m_{1}\right]$ by $f_{2}^{\prime}(i)=\hat{f}_{2}(i)$ for $i$ such that $\hat{f}_{2}(i)<m_{1}, f_{2}^{\prime}(i)=m_{1}$ for $\hat{f}_{2}(i) \geq m_{1}$, so that $\hat{D}_{2}$ follows $f_{2}^{\prime}$ in $D_{1}$. Further, $\left(\hat{D}_{2}, f_{2}^{\prime}\right)$ is compatible with $C_{1}$ relative to $\left(D_{1},\left\{d(1, j) \mid c\left(0, f_{1}(j)\right) \in X\right\}\right)$. Note that $p^{\prime} \in i\left(d\left(1, m_{1}\right), D_{1}\right)$. Let $\left\{d(1, j) \mid c\left(0, f_{1}(j)\right) \in X\right\}=X_{1}$ and let $X_{1}^{\prime}=\{d \in$ $\left.X_{1} \mid d \cap\left(D_{1}-X_{1}\right)^{*}=\varnothing\right\}$.

Finally, there is an open chain cover $D_{2}^{\prime}$ of $P$ such that $D_{2}^{\prime}=D_{2}^{\prime}\left[0, m_{2}^{\prime}\right]$ closure refines $\hat{D}_{2}, \hat{D}_{2}$ is an amalgamation of $D_{2}^{\prime}$ and $p^{\prime} \in i\left(d^{\prime}\left(2, m_{2}^{\prime}\right), D_{2}^{\prime}\right)$ with $d^{\prime}\left(2, m_{2}^{\prime}\right) \subseteq$ $i\left(d\left(1, m_{1}\right), D_{1}\right)$. Let $D_{2}=D_{2}\left[0, m_{2}\right]=\left\{\tilde{D}^{*} \mid \tilde{D}\right.$ is a maximal subchain of $D_{2}^{\prime}$ such that $\tilde{D}^{*} \subseteq d \in \hat{D}_{2}$ and $\tilde{D}^{*} \cap i\left(d, \hat{D}_{2}\right) \neq \varnothing$ for some $\left.d \in \hat{D}_{2}\right\}$. Without loss of generality, assume $p^{\prime} \in i\left(d\left(2, m_{2}\right), D_{2}\right)$. Then $D_{2}$ is an open chain cover of $P$ and $D_{2}$ follows the pattern $f_{2}$ in $D_{1}$, where $f_{2}(i)=f_{2}^{\prime}(j)$ when $d^{\prime}(2, j)$ is that unique link of $D_{2}^{\prime}$ such that $d(2, i) \subseteq d^{\prime}(2, j)$ and $d(2, i) \cap i\left(d^{\prime}(2, j), D_{2}^{\prime}\right) \neq \varnothing$, unless $d\left(1, f_{2}^{\prime}\right) \notin X_{1}^{\prime}, \overline{d(2, i)} \subseteq X_{1}^{\prime *}$, in which case $f_{2}(i)$ is defined so that $f_{2}$ prefers $X_{1}^{\prime}$. (We are applying Lemma 4 here.) We are done.

For $g, k \in H\left(P^{\prime}\right)$ define $\rho^{\prime}(g, k)=\operatorname{lub}\left\{d(g(x), k(x)) \mid x \in P^{\prime}\right\}$ and for $g, k \in H(P)$, define $\rho(g, k)=\operatorname{lub}\{d(g(x), k(x)) \mid x \in P\}$. Our strategy in the proof that follows will be the following. There is a sequence $u_{1}, u_{2}, \ldots$, of open sets of $P$ such that $\bar{u}_{1} \subseteq o, \overline{u_{i+1}} \subseteq u_{i}$ for $i \geq 1$, and $\bigcap u_{i}=P^{\prime}$. We will construct inductively a sequence $g_{1}, g_{2}, \ldots$ of homeomorphisms of $P$ so that

(1) $g_{1}(x)=x$ for $x \notin o$ and $g_{i+1}(x)=x$ for $x \notin g_{i} \cdot g_{i-1} \cdots \cdot g_{1}\left(u_{1}\right)$;

(2) $g_{i}\left(P^{\prime}\right)=P^{\prime}$ for each $i$;

(3) $\rho^{\prime}\left(g_{i} \mid P^{\prime}, h_{i}\right)<2^{-(i+1)}$ and $\rho^{\prime}\left(g_{i}^{-1} \mid P^{\prime}, h_{i}^{-1}\right)<2^{-(i+1)}$ for $i>1$, where $h_{i}=$ $h_{i-1} \cdot g_{i-1}^{-1} \mid P^{\prime} ;$ and

(4) $\rho\left(g_{i}, 1_{P^{\prime}}\right)<2^{-i}$ and $\rho\left(g_{i}^{-1}, 1_{P^{\prime}}\right)<2^{-i}$ for $i>1$, where $1_{P^{\prime}}$ denotes the identity on $P^{\prime}$.

We will then define $g(x)=\lim _{i} g_{i} \cdots \cdot g_{1}(x)$ and $g$ will be the homeomorphism we desired (as we later prove). 
PROOF OF THE THEOREM. Since the pseudoarc has the fixed point property, there is some $p^{\prime} \in P^{\prime}$ such that $h\left(p^{\prime}\right)=p^{\prime}$. Further, we may assume that there is some $q^{\prime} \in P^{\prime}$ such that $P^{\prime}$ is irreducible from $p^{\prime}$ to $q^{\prime}$ and $h\left(q^{\prime}\right)=q^{\prime}$. (If this is not the case, then we can extend $P^{\prime}$ and $h$ using Theorem LG, so that it is the case in the new continuum with the extended homeomorphism, and then prove the theorem for that homeomorphism and continuum.) Let us also assume, without loss of generality, that $q \in P-\bar{o}$ such that $q$ and $p^{\prime}$ are in different composants of $P$.

Suppose that $V_{1}, V_{2}, \ldots$ is a defining sequence for $P$ with the following properties:

(1) $\operatorname{mesh} V_{i}<2^{-i}$ for each $i$;

(2) for $i \in \mathbf{N}, V_{i}=\left\{v(i, 0), \ldots, v\left(i, n_{i}\right)\right\}$ with $q \in i\left(v(i, 0), V_{i}\right)$ and $p^{\prime} \in$ $i\left(v\left(i, n_{i}\right) V_{i}\right)$

(3) if $\overline{v(1, i)} \cap(P-o) \neq \varnothing$ and $\overline{v(1, j)} \cap P^{\prime} \neq \varnothing$, then $|j-i|>5$;

(4) for $i \geq 1$, mesh $V_{i+1} \leq \frac{1}{5} \min \left[\left\{d\left(v, v^{\prime}\right) \mid v, v^{\prime}\right.\right.$ are nonadjacent links of $\left.V_{i}\right\} \cup$ $\left\{d(x, v) \mid\left(x=p^{\prime}\right.\right.$ and $\left.v \neq v\left(i, n_{i}\right)\right)$ or $(x=q$ and $\left.\left.v \neq v(i, 0)), v \in V_{i}\right\}\right]$; and

(5) for $i \geq 1, q^{\prime} \in i\left(v\left(i, b_{i}^{\prime}\right), V_{i}\right)$, where $v\left(i, b_{i}^{\prime}\right)$ is the first link of $V_{i}$ whose closure intersects $P^{\prime}$. (To see that this last property can be satisfied, take a defining sequence with the other four properties, modify the chains involved using Lemma 3 (fattening and doubling in the subchains containing $P^{\prime}$ in their unions), and then doing any additional modifications to ensure that the result is a defining sequence with all five properties satisfied.)

Let $\varepsilon_{1}<\frac{1}{4}, \varepsilon_{1}>0$, such that if $\rho^{\prime}(k, 1)<\varepsilon_{1}$, then $\rho^{\prime}\left(k^{-1}, 1\right)<\frac{1}{4}$ for $k \in H\left(P^{\prime}\right)$. There is $\delta_{1}>0, \delta_{1}<\varepsilon_{1} / 2$, such that if $d(x, y)<\delta_{1}, x, y \in P^{\prime}$, then $d(h(x), h(y))<$ $\varepsilon_{1} / 2$ and $d\left(h^{-1}(x), h^{-1}(y)\right)<\varepsilon_{1} / 2$. Then there is $\tau_{1}>0, \tau_{1}<\delta_{1} / 2$, such that if $d(x, y)<\tau_{1}, x, y \in P^{\prime}$, then $d(h(x), h(y))<\delta_{1} / 2$ and $d\left(h^{-2}(x), h^{-2}(y)\right)<\delta_{1} / 2$. Also choose $\tau_{1}$ so that if $\rho^{\prime}(h, g)<\tau_{1}, \rho^{\prime}\left(h^{-1}, g^{-1}\right)<\delta_{1}$ for $g \in H\left(P^{\prime}\right)$, and choose $\tau_{1}^{\prime}>0, \tau_{1}^{\prime}<\tau_{1} / 2$ so that if $d(x, y)<\tau_{1}^{\prime}, x, y \in P^{\prime}$, then $d(h(x), h(y))<\tau_{1} / 2$.

Find $i_{1} \in \mathbf{N}$ such that mesh $V_{i_{1}}<\tau_{1}^{\prime}$. Let $V_{i_{1}}=C_{1}$, and let

$$
C_{1}=\left\{c(1,0), \ldots, c\left(1, m_{1}\right)\right\}
$$

where $p^{\prime} \in i\left(c\left(1, m_{1}\right), C_{1}\right), q \in i\left(c(1,0), C_{1}\right)$, and $q^{\prime} \in i\left(c\left(1, b_{1}\right), C_{1}\right)$. Then $C_{1}\left[b_{1}, m_{1}\right]$ essentially covers $P^{\prime}$, and $P-o \subseteq C_{1}\left[0, b_{1}-4\right]$.

For $i \in\left[b_{1}, m_{1}\right]$, let $c^{\#}(1, i)=c(1, i) \cap P^{\prime}$, and let $C_{1}^{\#}=\left\{c^{\#}(1, i) \mid i \in\left[b_{1}, m_{1}\right]\right\}$. Use Lemma 2 to obtain the open chains $E_{1}$ and $F_{1}$ as follows:

(1) Fatten $C_{1}^{\#}$ in $C_{1}\left[b_{1}, m_{1}\right]$, obtaining the open chain $\hat{E}_{1}=\hat{E}_{1}\left[b_{1}, m_{1}\right]$ so that $\overline{\hat{E}}_{1}^{*} \cap \overline{C_{1}\left[0, b_{1}-1\right]^{*}}=\varnothing$ and mesh $\hat{E}_{1}<\tau_{1}^{\prime}$.

(2) Fatten $h\left(C_{1}^{\#}\right)=\left\{h\left(c^{\#}\right) \mid c^{\#} \in C_{1}^{\#}\right\}$ in $\hat{E}_{1}^{*}$ obtaining the open chain $F_{1}=$ $F_{1}\left[b_{1}, m_{1}\right]$.

(3) Let $E_{1}=E_{1}\left[b_{1}, m_{1}\right]$ be defined by $e(1, i)=\hat{e}(1, i) \cap F_{1}^{*}$ for $i \in\left[b_{1}, m_{1}\right]$.

(4) Further, do these fattenings so that $\partial E_{1}^{*} \subseteq j\left(e\left(1, m_{1}\right), E_{1}\right) \subseteq i\left(c\left(1, m_{1}\right), C_{1}\right)$ and $\partial F_{1}^{*} \subseteq j\left(f\left(1, m_{1}\right), F_{1}\right)$. (Note $E_{1}^{*}=F_{1}^{*}$.)

We are almost ready to construct $D_{1}$. Let us make one more assumption: The links in $F_{1}$ may not be $V_{j}$-open for any $j$, although we can construct $E_{1}$ so that those links are $V_{j}$-open for some $j$. However, with an arbitrarily small enlargement of each link, we can make the links of $F_{1} V_{k}$-open for some $k$. Of course, it will no longer necessarily be the case that $h\left(c^{\#}(1, i)\right)=f(1, i) \cap P^{\prime}$ for $i \in\left[b_{1}, m_{1}\right]$. We 
assume then that the links of $F_{1}$ and of $E_{1}$ are all $V_{k}$-open for some $k$. Let

$$
\begin{aligned}
D_{1}= & \left\{c(1,0), c(1,1), \ldots, c\left(1, b_{1}-1\right),\right. \\
& \left.\left(c\left(1, b_{1}\right)-F_{1}^{*}\right) \cup f\left(1, b_{1}\right), \ldots,\left(c\left(1, m_{1}\right)-F_{1}^{*}\right) \cup f\left(1, m_{1}\right)\right\} \\
= & D_{1}\left[0, m_{1}\right]=\left\{d(1,0), \ldots, d\left(1, m_{1}\right)\right\},
\end{aligned}
$$

and let $X_{1}=C_{1}\left[0, b_{1}-1\right]=D_{1}\left[0, b_{1}-1\right]$. Choose $i_{2} \in \mathbf{N}$ so that $i_{2}>i_{1}$, the links of both $D_{1}$ and $C_{1}$ are $V_{i_{2}}$-open, and mesh $V_{i_{2}}<\tau_{2}$ where (a) $\varepsilon_{2}>0$ such that $\varepsilon_{2}<1 / 8, \varepsilon_{2}<\tau_{1}^{\prime}$, (b) $\delta_{2}>0, \delta_{2}<\varepsilon_{2} / 2$, such that if $x, y \in P^{\prime}$ and $d(x, y)<\delta_{2}$, then $d(h(x), h(y))<\varepsilon_{2} / 2$ and $d\left(h^{-1}(x), h^{-1}(y)\right)<\varepsilon_{2} / 2$, (c) $\tau_{2}>0, \tau_{2}<\delta_{2} / 2$ such that if $x, y \in P^{\prime}$ and $d(x, y)<\tau_{2}$, then $d(h(x), h(y))<\delta_{2} / 2$ and (d) $V_{i_{2}}$ closure refines $C_{1}$ and $D_{1}$. Also, choose $i_{2}$ so that mesh $V_{i_{2}}<\frac{1}{4} \min \left\{d\left(v, v^{\prime}\right) \mid\right.$ for $i \in\left[0, m_{1}-1\right]$, $v=c(1, i)-E_{1}^{*}, v^{\prime}=E_{1}^{*}$ or $\left.\left.v=d(1, i)-F_{1}^{*}, v^{\prime}=F_{1}^{*}\right\}, \overline{v\left(i_{2}, n_{i_{2}}\right.}\right) \subseteq i\left(d\left(1, m_{1}\right), D_{1}\right)$, and $\operatorname{mesh} V_{i_{2}}<\frac{1}{6} \min \left\{d\left(p^{\prime}, e\right) \mid p^{\prime} \notin e, e \in C_{1} \cup D_{1}\right\}$.

Let $C_{2}=\left\{c(2,0), \ldots, c\left(2, m_{2}\right)\right\}=V_{i_{2}}$ where $q \in c(2,0), p^{\prime} \in c\left(2, m_{2}\right), q^{\prime} \in$ $c\left(2, b_{2}\right)$. Now $C_{2}$ follows some pattern $f_{1}:\left[0, m_{2}\right] \rightarrow\left[0, m_{1}\right]$ in $C_{1}$ where $f_{1}$ prefers $X_{1}$. Then $\left(C_{2}, f_{1}\right)$ is compatible with $D_{1}$ relative to $\left(C_{1}, X_{1}\right)$, and we can apply Lemma 5 . Choose $\sigma_{2}<\frac{1}{16}, \sigma_{2}>0, \sigma_{2}<\tau_{2}, \sigma_{2}<$ Lebesgue number for $\left\{h\left(c \cap P^{\prime}\right) \mid c \in C_{2}\left[b_{2}, m_{2}\right]\right\}$. Then there exist chains $\hat{D}_{2}$ and $\hat{D}_{3}$ covering $P$ so that $\hat{D}_{2}$ follows $f_{1}$ in $D_{1}$, mesh $\hat{D}_{3}<\sigma_{2}, \hat{D}_{3}$ follows a pattern $\hat{f}_{2}$ in $\hat{D}_{2}, p^{\prime} \in$ $i\left(\hat{d}\left(2, m_{2}\right), \hat{D}_{2}\right) \cap i\left(\hat{d}\left(3, \hat{m}_{3}\right), \hat{D}_{3}\right)$, and $\left(\hat{D}_{3}, \hat{f}_{2}\right)$ is compatible with $C_{2}$ relative to $\left(\hat{D}_{2}, X_{2}^{\prime}\right)$ (where $\hat{D}_{2}=\hat{D}_{2}\left[0, m_{2}\right], X_{2}=\left\{c(2, j) \mid c\left(1, f_{1}(j)\right) \in X_{1}^{\prime}\right\}$ and $X_{2}^{\prime}=\{c \in$ $\left.\left.X_{2} \mid c \cap\left(C_{2}-X_{2}\right)^{*}=\varnothing\right\}\right)$.

Without loss of generality assume that $\hat{D}_{2}$ and $\hat{D}_{3}$ are $V_{l}$-open for some $l$. We need for $q^{\prime}$ to be in $i\left(\hat{d}\left(2, b_{2}\right), \hat{D}_{2}\right)$, but this may not be the case. We can, however, modify $\hat{D}_{2}$ and $\hat{D}_{3}$ so that this will be the case, without losing the other properties we want these chains to have.

Let $D_{2}^{\#}=\left\{h\left(c \cap P^{\prime}\right) \mid c \in C_{2}, c \cap P^{\prime} \neq \varnothing\right\}$. Fatten $D_{2}^{\#}$ (using Lemma 2) and then enlarge the links slightly to obtain the chain $F_{2}$ with the end link exit property so that

(1) $F_{2}=\left\{f(2, i) \mid h\left(c(2, i) \cap P^{\prime}\right) \subseteq f(2, i) \cap P^{\prime}, i \in\left[b_{2}, m_{2}\right]\right\}$;

(2) $\partial F_{2}^{*} \subseteq i\left(\hat{d}\left(2, m_{2}\right), \hat{D}_{2}\right) \cap i\left(c\left(2, m_{2}\right), C_{2}\right) \cap j\left(f\left(2, m_{2}\right), F_{2}\right)$;

(3) $F_{2}^{*}$ is $V_{j}$-open for some $j$;

(4) $F_{2}^{*} \subseteq C_{2}\left[b_{2}, m_{2}\right]^{*} \cap \tilde{D}_{3}^{*}$, where $\tilde{D}_{3}$ is that unique subchain of $\hat{D}_{3}$ such that $\tilde{D}_{3}^{*}$ contains $P^{\prime}$ and each link of $\tilde{D}_{3}$ intersects $P^{\prime}$ and

(5) $\overline{f(2, i)} \subseteq f\left(1, f_{1}(i)\right)$ for $i \in\left[b_{2}, m_{2}\right]$.

Let $D_{2}=\left\{\left(\hat{d}(2, i)-F_{2}^{*}\right) \cup f(2, i) \mid i \in\left[0, m_{2}\right]\right\}$, where we define $f(2, i)=\varnothing$ for $i \in\left[0, b_{2}-1\right]$, so that $D_{2}$ is an open chain cover of $P$ so that $D_{2}$ follows $f_{1}$ in $D_{1}$, $q^{\prime} \in i\left(d\left(2, b_{2}\right), D_{2}\right), p^{\prime} \in i\left(d\left(2, m_{2}\right), D_{2}\right)$, and $q \in i\left(d(2,0), D_{2}\right)$.

Find $i_{3}$ so that $V_{i_{3}}$ closure refines $\hat{D}_{3}, D_{2}, \hat{D}_{2}$, and $C_{2}$; each of $\hat{D}_{3}, D_{2}, \hat{D}_{2}$ and $C_{2}$ is an amalgamation of $V_{i_{3}}$; and no link of $V_{i_{3}}$ intersects both $\hat{d}(2, i)-F_{2}^{*}$ and $F_{2}^{*}$ for some $i<m_{2}$. If $D_{3}=D_{31} \cup D_{32}$ where $D_{31}=\left\{\tilde{D}^{*} \mid \tilde{D}\right.$ is a maximal subchain of $V_{i_{3}}$ such that (1) $\tilde{D}^{*} \subseteq d$ for some $d \in \hat{D}_{3},(2)$ no closure of a link of $\tilde{D}$ is in $F_{2}^{*}$, and (3) $\left.\tilde{D}^{*} \cap i\left(d, \hat{D}_{3}\right) \neq \varnothing\right\}$, and $D_{32}=\left\{d \in V_{i_{3}} \mid d \subseteq F_{2}^{*}\right\}$, then $D_{3}=D_{3}\left[0, m_{3}\right]$ is an open chain cover of $P$ and without loss of generality we can assume that $p^{\prime} \in$ $i\left(d\left(3, m_{3}\right), D_{3}\right)$. It will follow that $q^{\prime} \in i\left(d\left(3, b_{3}\right), D_{3}\right)$ where $D_{3}\left[b_{3}, m_{3}\right]$ essentially 
covers $P^{\prime}$, no proper subchain of $D_{3}\left[b_{3}, m_{3}\right]$ covers $P^{\prime}$, and $\overline{D_{3}\left[0, b_{3}-1\right]^{*}} \cap P^{\prime}=\varnothing$. Then $D_{3}$ follows the pattern $f_{2}$ in $D_{2}$ where we define $f_{2}(i)=\hat{f}_{2}(j)$ where $j$ is that unique integer such that $\hat{d}(3, j) \supseteq d(3, i)$ and $d(3, i) \cap i\left(\hat{d}(3, j), \hat{D}_{3}\right) \neq \varnothing,(1)$ unless $\overline{d(3, i)} \subseteq X_{2}^{\prime *}$, but $d\left(2, \hat{f}_{2}(j)\right) \notin X_{2}^{\prime}$, in which case we define $f_{2}$ so that $f_{2}$ prefers $X_{2}^{\prime}$, or, $(2) d(3, i) \subseteq F_{2}^{*}$, in which case $f_{2}(i)=k$ where $f(2, k)$ is some link of $F_{2}$ such that $f(2, k) \supseteq \underline{\hat{d}(3, i)}$. It will follow that $\left(D_{3}, f_{2}\right)$ is compatible with $C_{2}$ relative to $\left(D_{2}, X_{2}^{\prime}\right)$.

Continue this process. Choose $\sigma_{3}>0, \sigma_{3}<\sigma_{2}, \sigma_{3}<1 / 32, \sigma_{2}<$ Lebesgue number for $\left\{h^{-1}\left(d \cap P^{\prime}\right) \mid d \in D_{3}\left[b_{3}, m_{3}\right]\right\}$. There exist chains $C_{3}=C_{3}\left[0, m_{3}\right]$ and $C_{4}=C_{4}\left[0, m_{4}\right]$ so that (1) mesh $C_{4}<\sigma_{3} ;$ (2) $C_{4}$ follows a pattern $f_{3}$ in $C_{3}$; (3) $C_{3}$ follows $f_{2}$ in $C_{2}$; (4) $f_{3}$ prefers $X_{3}^{\prime}=\left\{c \in X_{3} \mid c \cap\left(C_{3}-X_{3}\right)^{*}=\varnothing\right\}$, where $X_{3}=\left\{c(3, j) \mid c\left(2, f_{2}(j)\right) \in X_{2}^{\prime}\right\} ;(5)\left(C_{4}, f_{3}\right)$ is compatible with $D_{3}$ relative to $\left(C_{3}, X_{3}^{\prime}\right)$; and (6)(a) $q \in i\left(c(3,0), C_{3}\right) \cap i\left(c(4,0), C_{4}\right)$, (b) $q^{\prime} \in i\left(c\left(3, b_{3}\right), C_{3}\right) \cap$ $i\left(c\left(4, b_{4}\right), C_{4}\right)\left(b_{4}\right.$ being that unique integer such that $C_{4}\left[b_{4}, m_{4}\right]$ essentially covers $P^{\prime}$ and $\left.P^{\prime} \cap \overline{C_{4}\left[0, b_{4}-1\right]^{*}}=\varnothing\right)$, and (c) $p^{\prime} \in i\left(c\left(3, m_{3}\right), C_{3}\right) \cap i\left(c\left(4, m_{4}\right), C_{4}\right)$. Having all this, we continue, choosing $D_{4}, D_{5}$ accordingly, etc., obtaining sequences $C_{1}$, $C_{2}, \ldots$, and $D_{1}, D_{2}, \ldots$, of open chain covers of $P$, and a sequence $f_{1}, f_{2}, \ldots$, of patterns such that

(1) $C_{i}=\left\{c(i, 0), \ldots, c\left(i, m_{i}\right)\right\}$, and $D_{i}=\left\{d(i, 0), \ldots, d\left(i, m_{i}\right)\right\}$ such that $q \in$ $c(i, 0)=d(i, 0), q^{\prime} \in i\left(c\left(i, b_{i}\right), C_{i}\right) \cap i\left(d\left(i, b_{i}\right), D_{i}\right)$, and

$$
p^{\prime} \in i\left(d\left(i, m_{i}\right), D_{i}\right) \cap i\left(c\left(i, m_{i}\right), C_{i}\right) ;
$$

(2) $C_{i+1}$ follows $f_{i}$ in $C_{i}$ and $D_{i+1}$ follows $f_{i}$ in $D_{i}$;

(3) $f_{i}$ prefers $X_{i}^{\prime} \subseteq C_{i} \cap D_{i}$ and $P-o \subseteq X_{i}^{\prime *}$; and

(4) $\lim _{i} \operatorname{mesh} C_{i}=0=\lim _{i} \operatorname{mesh} D_{i}$.

Thus, by Theorem 6 of $[\mathbf{K R}]$, we have induced a homeomorphism $g_{1} \in H(P)$ defined by $g_{1}(x)=\bigcap_{i=1}^{\infty} d(i, j(x, i))$ where for each $i, x \in c(i, j(x, i))$ and $f_{i-1}(j(x, i))$ $=j(x, i-1)(i>1)$. By construction $g_{1}(x)=x$ for $x \notin o$ and $g_{1}\left(p^{\prime}\right)=p^{\prime}$, $g_{1}\left(q^{\prime}\right)=q^{\prime}$. Also, since $P^{\prime}$ is irreducible from $p^{\prime}$ to $q^{\prime}, g_{1}\left(P^{\prime}\right)=P^{\prime}$. Thus, if $x \in P^{\prime}$, there is an infinite sequence $j(x, 1), j(x, 2), \ldots$, of integers such that for $i \geq 2$, $x \in c(i, j(x, i))$ and $f_{i-1}(j(x, i))=j(x, i-1)$, and, in particular, $x \in c(1, j(x, 1))$ and $g_{1}(x) \in d(1, j(x, 1))=\left(c(1, j(x, 1))-F_{1}^{*}\right) \cup f(1, j(x, 1))$. Hence, $g_{1}(x) \in f(1, j(x, 1))$, $x \in e(1, j(x, 1))$, and $h(x) \in f(1, j(x, 1))$. Then $d\left(g_{1}(x), h(x)\right)<\tau_{1}\left(\operatorname{mesh} F_{1}<\tau_{1}\right.$ by construction). Likewise $d\left(g_{1}^{-1}(x), h^{-1}(x)\right)<\delta_{1}$, and $\rho^{\prime}\left(1_{P^{\prime}}, h g_{1}^{-1} \mid P^{\prime}\right)<\varepsilon_{1}$, $\rho^{\prime}\left(1_{P^{\prime}}, g_{1} h^{-1}\right)<\frac{1}{4}$. (Let $1_{P^{\prime}}$ denote the identity of $P^{\prime}$.) Now we just continue this process. Let $h_{2}=h g_{1}^{-1} \mid P^{\prime} \in H\left(P^{\prime}\right)$, and choose $\rho_{2}>0$ such that $\rho_{2}>\rho^{\prime}\left(1_{P^{\prime}}, h_{2}\right)$ and $\rho_{2}>\rho^{\prime}\left(1_{P^{\prime}}, h_{2}^{-1}\right), \rho_{2}<\frac{1}{4}$ (unless $h_{2}=g_{1} \mid P^{\prime}$, in which case we are done). There are $\tau_{21}^{\prime}, \tau_{21}, \delta_{21}, \varepsilon_{21}>0$ such that $\frac{1}{8}$

(a) $\varepsilon_{21}>\frac{1}{2}\left(\frac{1}{4}-\rho_{2}\right), \rho_{2}, \frac{1}{8}$ and if $\rho^{\prime}\left(l, h_{2}\right)<\varepsilon_{21}$ for $l \in H\left(P^{\prime}\right)$, then $\rho\left(l^{-1}, h_{2}^{-1}\right)<$

(b) $\delta_{21}<\varepsilon_{21} / 2$ and if $x, y \in P^{\prime}, d(x, y)<\delta_{21}$, then

$$
d\left(h_{2}(x), h_{2}(y)\right), d\left(h_{2}^{-1}(x), h_{2}^{-1}(y)\right), d\left(h_{2}^{-2}(x), h_{2}^{-2}(y)\right)<\varepsilon_{21} / 2 ;
$$

(c) $\tau_{21}<\delta_{21} / 2$ and if $x, y \in P^{\prime}, d(x, y)<\tau_{21}$, then

$$
d\left(h_{2}(x), h_{2}(y)\right), d\left(h_{2}^{-1}(x), h_{2}^{-1}(y)\right), d\left(h_{2}^{-2}(x), h_{2}^{-2}(y)\right)<\delta_{21} / 2 ;
$$

(d) if $l \in H\left(P^{\prime}\right)$ and $\rho^{\prime}\left(h_{2}, l\right)<\tau_{21}, \rho\left(h_{2}^{-1}, l^{-1}\right)<\delta_{21}$; and 
(e) $\tau_{21}^{\prime}<\tau_{21} / 2$ and if $x, y \in P^{\prime}, d(x, y)<\tau_{21}^{\prime}$, then

$$
d\left(h_{2}(x), h_{2}(y)\right), d\left(h_{2}^{-1}(x), h_{2}^{-1}(y)\right), d\left(h_{2}^{-2}(x), h_{2}^{-2}(y)\right)<\tau_{21} / 2 .
$$

There is a sequence $u_{1}, u_{2}, \ldots$, of open sets of $P$ such that $\bar{X}_{1}^{*} \cap \bar{u}_{1}=\varnothing, P^{\prime} \subseteq$ $\ldots \subseteq \bar{u}_{3} \subseteq u_{2} \subseteq \bar{u}_{2} \subseteq u_{1} \subseteq \bar{u}_{1}, P^{\prime}=\bigcap_{i} u_{i}$. Find $i_{21} \in \mathbf{N}$ such that mesh $V_{i_{21}}<\tau_{21}^{\prime}$, mesh $V_{i_{21}}<\mu_{1}$, where $\overline{D_{\mu_{1}}\left(P^{\prime}\right)} \subseteq E_{1}^{*}$, and if $\tilde{C}_{1}$ denotes the maximal subchain of $V_{i_{21}}$ with the property that the closure of each link of $\tilde{C}_{1}$ intersects $P^{\prime}$, then $\overline{\tilde{C}_{1}^{*}} \cap\left(g_{1}\left(P-u_{1}\right)\right)=\varnothing$ and there are at least five links of $V_{i_{21}}$ between any link in $\tilde{C}_{1}$ and any link intersecting $g_{1}\left(P-u_{1}\right)$. Let $C_{21}=V_{i_{21}}$ and from here, with $X_{21}=\left\{c \in C_{21} \mid c \notin \tilde{C}_{1}\right\}$, construct a stable homeomorphism $g_{2} \in H(P)$ such that $g_{2}(x)=x$ for $x \in g_{1}\left(P-u_{1}\right), g_{2}\left(p^{\prime}\right)=p^{\prime}$ and $g_{2}\left(q^{\prime}\right)=q^{\prime}$. Further construct $g_{2}$ so that $\rho^{\prime}\left(1_{P^{\prime}}, h_{2} g_{2}^{-1} \mid P^{\prime}\right)<\frac{1}{8}, \rho^{\prime}\left(1_{P^{\prime}}, g_{2} h_{2}^{-1}\right)<\frac{1}{8}$, and $\rho\left(1_{P}, g_{2}\right)<\frac{1}{4}, \rho\left(1_{P}, g_{2}^{-1}\right)<\frac{1}{4}$. Note that if $x \in P-u_{1}, g_{2} g_{1}(x)=g_{1}(x)$. Continue, obtaining a sequence $g_{1}, g_{2}, \ldots$, of stable homeomorphisms such that for each $i$

(1) $g_{i}(x)=x$ for $x \in g_{i-1} \cdot g_{i-2} \cdots \cdot g_{1}\left(P-u_{i-1}\right)$;

(2) $g_{i}\left(p^{\prime}\right)=p^{\prime}, g_{i}\left(q^{\prime}\right)=q^{\prime}$

(3) $\rho\left(1, g_{i}\right)<2^{-i}$ and $\rho\left(1, g_{i}^{-1}\right)<2^{-i}$ (unless $i=1$ );

(4) $\rho^{\prime}\left(1_{P^{\prime}}, h_{i} g_{i}^{-1} \mid P^{\prime}\right)<2^{-(i+1)}, \rho^{\prime}\left(1_{P^{\prime}}, g_{i} h_{i}^{-1}\right)<2^{-(i+1)}$, for $h_{i}=h_{i-1} g_{i-1}^{-1} \mid P^{\prime}$ $(i>2)$.

Define $g: P \rightarrow P$ by $g=\lim _{i} g_{i} \cdots g_{1}$. By construction, $g$ is a continuous function from $P$ onto $P$ such that $g\left(P^{\prime}\right)=P^{\prime}$. Futher, if $x \notin P^{\prime}, x \in P-U_{i}$ for some $i$, and $g(x)=g_{i} \cdots g_{1}(x) \notin P^{\prime}$. Thus, $g$ is one-to-one on $P-P^{\prime}$. If $x \in P^{\prime}$,

$$
\begin{gathered}
d\left(x, g_{1} h^{-1}(x)\right)<\frac{1}{4}, \quad d\left(x, g_{2} h_{2}^{-1}(x)\right)=d\left(x, g_{2} g_{1} h^{-1}(x)\right)<\frac{1}{8}, \\
d\left(x, g_{3} h_{3}^{-1}(x)\right)=d\left(x, g_{3} g_{2} g_{1} h^{-1}(x)\right)<\frac{1}{16}, \quad \text { etc. }
\end{gathered}
$$

Then $g_{1} h^{-1}(x), g_{2} g_{1} h^{-1}(x), \ldots$, converges to $x$ for $x \in P^{\prime}$, and, thus, $g h^{-1}$ is a homeomorphism on $P^{\prime}$, and, in fact, $1_{P^{\prime}}$. So, $g \mid P^{\prime}=h$ and $g$ is one-to-one on all of $P$, and $g$ is an extension of $h$. We have the homeomorphism we desired.

COROLLARY 1. If $P^{\prime}$ is a proper subcontinuum of $X$, a continuum with the property that each of its nondegenerate proper subcontinua is a pseudoarc, $h^{\prime}$ is a homeomorphism from $P^{\prime}$ onto itself, and $o$ is a open set in $P$ such that $P^{\prime} \subseteq o$, then there is a homeomorphism $h \in H(P)$ such that $h \mid P^{\prime}=h^{\prime}$ and $h(x)=x$ for $x \notin o$.

PROOF. This follows from Theorem 8 of $[\mathbf{K J}]$ and our main theorem.

COROLLARY 2. Suppose that (1) $n_{1}, n_{2}, \ldots$, is a sequence of positive integers, (2) $o_{1}, o_{2}, \ldots$, is a sequence of disjoint open sets in the continuum $X$ with the property that each of its nondegenerate proper subcontinua is a pseudoarc, and (3) for $i \in \mathbf{N}, P_{i}$ is a nondegenerate subcontinuum contained in $o_{i}$ with $x_{i} \in P_{i}$. Then there is a homeomorphism $h \in H(X)$ such that for each $i \in \mathbf{N}, h\left(P_{i}\right)=P_{i}, x_{i}$ has period $n_{i}$ under $h$, and $h(x)=x$ for $x \notin \bigcup o_{i}$.

PROOF. Use Theorem LG to find $h_{i} \in H\left(P_{i}\right)$ such that $x_{i}$ has period $n_{i}$, and then extend $h_{i}$ to $\hat{h}_{i} \in H(P)$ such that $\hat{h}_{i}(x)=x$ for $x \notin o_{i}$ and $\hat{h}_{i} \mid P_{i}=h_{i}$. Then define

$$
h(x)= \begin{cases}h_{i}(x) & \text { if } x \in o_{i} \text { for some } i \\ x & \text { otherwise }\end{cases}
$$




\section{REFERENCES}

[B] R. H. Bing, Snake-like continua, Duke Math. J. 18 (1951), 653-663.

[C] Howard Cook, Concerning three questions of Burgess about homogeneous continua, Colloq. Math. 19 (1968), 241-244.

[F] L. Fearnley, Hereditarily indecomposable circularly chainable continua, Ph. D. Thesis, University of London, 1970.

[H] O. H. Hamilton, A fixed point theorem for pseudoarcs and certain other metric continua, Proc. Amer. Math. Soc. 2 (1951), 173-174.

[I] W. T. Ingram, Hereditarily indecomposable tree-like continua, Fund. Math. 103 (1979), 61-64.

[KJ] Judy Kennedy, Continua whose proper subcontinua are chainable, Topology Proc. 10 (1985), $111-134$.

[KR] Judy Kennedy and James T. Rogers, Jr., Orbits of the pseudocircle, Trans. Amer. Math. Soc. 296 (1986), 327-340.

[KB] B. Knaster, In continu dont tout sous-continu est indécomposable, Fund. Math. 3 (1922), $247-286$.

[LG] G. R. Lehner, Extending homeomorphisms on the pseudo-arc, Trans. Amer. Math. Soc. 98 (1961), 369-394.

[LW] Wayne Lewis, Stable homeomorphisms of the pseudo-arc, Canad. J. Math. 31 (1979), 363-374.

[OT] Lex G. Oversteegen and E. D. Tymchatyn, On hereditarily indecomposable compacta, Geometric and Algebraic Topology, Banach Centre Publ., 18, PWN, Warsaw, 1986, pp. 403-413.

Department of Foundations, Analysis, and Topology, Division of Mathematics, Auburn University, Auburn, Alabama 36849

Department of Mathematical Sciences, University of Delaware, Newark, DELAWARE 19716 